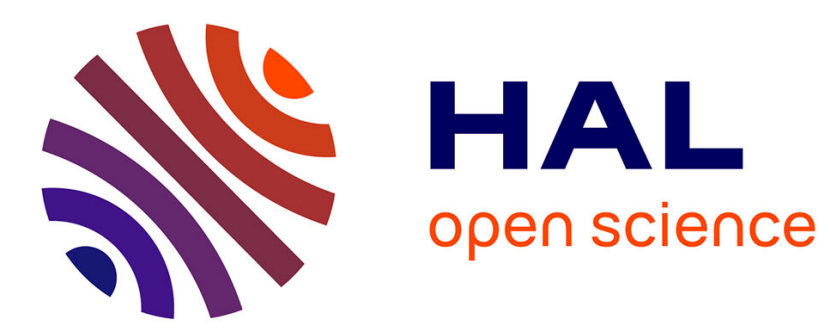

\title{
Degenerate Poisson Distribution
}

Ugur Duran, Mehmet Acikgoz

\section{- To cite this version:}

| Ugur Duran, Mehmet Acikgoz. Degenerate Poisson Distribution. 2019. hal-02372338

\section{HAL Id: hal-02372338 \\ https://hal.science/hal-02372338}

Preprint submitted on 20 Nov 2019

HAL is a multi-disciplinary open access archive for the deposit and dissemination of scientific research documents, whether they are published or not. The documents may come from teaching and research institutions in France or abroad, or from public or private research centers.
L'archive ouverte pluridisciplinaire HAL, est destinée au dépôt et à la diffusion de documents scientifiques de niveau recherche, publiés ou non, émanant des établissements d'enseignement et de recherche français ou étrangers, des laboratoires publics ou privés. 


\title{
Degenerate Poisson Distribution
}

\author{
Ugur Duran ${ }^{1, *}$ and Mehmet Acikgoz ${ }^{2}$ \\ ${ }^{1}$ Department of the Basic Concepts of Engineering, \\ Faculty of Engineering and Natural Sciences, \\ Iskenderun Technical University, TR-31200 Hatay, Turkey \\ E-Mail: mtdrnugur@gmail.com8\} ugur.duran@iste.edu.tr \\ ${ }^{*}$ Corresponding Author \\ ${ }^{2}$ University of Gaziantep, Faculty of Science and Arts, \\ Department of Mathematics, TR-27310 Gaziantep, Turkey \\ E-Mail: acikgoz@gantep.edu.tr
}

\begin{abstract}
The main purpose of this paper is to introduce and investigate degenerate Poisson distribution which is a new extension of the Poisson distribution including the degenerate exponential function. We then provide several properties of the degenerate Poisson distribution such as the first and the second raw moments and difference operator property. Moreover, we acquired the skewness and the kurtosis for the degenerate Poisson distribution. We also derive its moment generating function by which we define the degenerate Bell polynomials and give a connection for these polynomials related to the unsigned Stirling numbers of the first kind.
\end{abstract}

2010 Mathematics Subject Classification. Primary 60E05; Secondary 11B73, 33B10.

Key Words and Phrases. Poisson distribution; raw moments; Bell polynomials; degenerate exponential function; unsigned Stirling number of the first kind.

\section{INTRODUCTION}

Probability distributions possess many importance and have been commonly used in statistics and probability theory. They can be given by a table or an equation which links each outcome of a statistical experiment with its probability of occurrence, see $[5,6,7,11,12]$ for more information about the topic on probability distribution. One of the most useful and applicable distribution is the Poisson probability distribution ( $c f$. $[5,6,7,11,12])$. The Poisson distribution is a discrete probability distribution that expresses the probability of a given number of events occurring in a fixed interval of time or space if these events occur with a known constant rate and independently of the time since the last event. The Poisson distribution can be also utilized for the number of events in other specified intervals such as volume, area or distance, $c f .[5,6,7,11,12]$ and see also the references cited therein.

Several generalizations of the Poisson distribution such as the Polya process, truncation of the domain, stuttering Poisson distribution, linear combinations of Poisson variables, particle counting, collective risk theory, mixed Poisson distributions, Erlang process and Poisson mixing distributions have been deeply discussed in the book [6] in 1967. In recent years, some scientists have been considered several new extensions of the Poisson distribution ( $c f$. [7, 11, 12]). For instance, Hermann [7] considered a generalization of the Poisson distribution based on the generalized Mittag-Leffler function and calculated the corresponding raw moments algebraically in terms of the familiar Bell polynomials. Mahmoudi [11] introduced the exponentiated Weibull-Poisson (EWP) distribution which includes four parameters as increasing, decreasing, bathtubshaped and unimodal failure rate and satisfied diverse properties for this distribution covering moments, quantiles, its probability density function and its reliability and failure rate functions. Porwal [12] defined Mittag-Leffler type Poisson distribution and then provided several properties of this distribution. In this 
paper, we consider a novel extension for the Poisson distribution which includes the degenerate exponential function and we call it the degenerate Poisson distribution. Then, we investigate multifarious properties of the degenerate Poisson distribution covering the first and the second raw moments. We also attained the skewness and the kurtosis for this distribution. Furthermore, we derive its moment generating function by which we consider the degenerate Bell polynomials and present a connection for the mentioned polynomials associated with the unsigned Stirling numbers of the first kind.

Here we provide some basic information taken from the references $[5,6,7,11,12]$.

Definition 1. The $n$-th moment of a discrete probability distribution about $X=0$ is defined by

$$
\mu_{n}=E\left(X^{n}\right)
$$

and the moment generating function (m.g.f.) of a random variable $X$ is denoted by $M_{X}(t)$ and defined by

$$
M_{X}(t)=E\left(e^{t X}\right)
$$

The probability density function of Poisson distribution with parameter $\lambda$ is defined as follows

$$
p_{x}(\lambda)=P(X=x)=\frac{1}{N} \frac{\lambda^{x}}{x !} \quad \text { for } x=0,1,2, \ldots,
$$

where $N$ is the normalization constant and is determined by the requirement of normalizability of the distribution, which coincides with the zeroth raw moment $\mu_{0}$ :

$$
\mu_{0}=\sum_{x=0}^{\infty} p_{x}(\lambda)=\sum_{x=0}^{\infty} \frac{1}{N} \frac{\lambda^{x}}{x !}=\frac{1}{N} \sum_{x=0}^{\infty} \frac{\lambda^{x}}{x !}=\frac{1}{N} e^{\lambda}=1
$$

which implies $N=e^{\lambda}$.

The first and the second raw moments for the Poisson distribution are given below:

$$
\mu_{0}=E(X)=\lambda \text { and } \mu_{1}=E\left(X^{2}\right)=\lambda+\lambda^{2},
$$

therefore, we obtain the mean $E(X)$ and the variance $V(X)$ of the Poisson distribution

$$
\text { (Mean) } E(X)=\lambda \text { and (Variance) } V(X)=\lambda \text {. }
$$

The moment generating function of the Poisson distribution is as follows:

$$
E\left(e^{x t}\right)=\sum_{x=0}^{\infty} e^{x t} p_{x}(\lambda)=e^{-\lambda} \sum_{x=0}^{\infty} \frac{\left(e^{t} \lambda\right)^{x}}{x !}=e^{\lambda\left(e^{t}-1\right)}=\sum_{n=0}^{\infty} B_{n}(\lambda) \frac{t^{n}}{n !},
$$

which is the generating function of the classical Bell polynomials $B_{n}(\lambda)(c f$. [2,7-10]). So, for $n \geq 0$, we write the higher raw moments by means of the Bell polynomials as follows:

$$
\mu_{n}=E\left(X^{n}\right)=B_{n}(\lambda) .
$$

A more detailed information about the topics mentioned above can be found in $[5,6,7,11,12]$ and each of the related references cited therein.

\section{Degenerate Poisson Distribution}

In this section, we consider an extension for the Poisson distribution via the degenerate exponential function $e_{\alpha}^{x}(t)$ and the $\alpha$-falling factorial $(x)_{n, \alpha}$. We call it the degenerate Poisson distribution. We then perform to obtain the first few raw moments and the moment generating function for the aforesaid distribution. By means of this moment generating function, we define the degenerate Bell polynomials and find the properties for these polynomials.

For $\alpha$ being a real number, the $\alpha$-falling factorial $(x)_{n, \alpha}$ is defined by (see [3, 4])

$$
(x)_{n, \alpha}= \begin{cases}x(x-\alpha)(x-2 \alpha) \cdots(x-(n-1) \alpha), & n=1,2, \ldots \\ 1, & n=0 .\end{cases}
$$

The degenerate exponential function $e_{\alpha}^{x}(t)$ for a real number $\alpha$ is given by $(c f .[4,9,10])$

$$
e_{\alpha}^{x}(t)=(1+\alpha t)^{\frac{x}{\alpha}} \quad \text { and } \quad e_{\alpha}^{1}(t)=e_{\alpha}(t) .
$$


It is readily seen that $\lim _{\alpha \rightarrow 0} e_{\alpha}^{x}(t)=e^{x t}(c f .[4,9,10])$. From (2.1) and (2.2), the degenerate exponential function possesses the following series expansion

$$
e_{\alpha}^{x}(t)=\sum_{n=0}^{\infty}(x)_{n, \alpha} \frac{t^{n}}{n !}
$$

We now introduce a generalization of the Poisson distribution via the $\alpha$-falling factorial $(x)_{n, r}$ as follows:

$$
p_{x}(\lambda, \alpha)=P(X=x)=\frac{1}{N} \frac{(\lambda)_{x, \alpha}}{x !} \quad \text { for } \quad x=0,1,2, \ldots
$$

the normalization constant $N$ follows from the requirement of normalizability of the distribution:

$$
\mu_{0}=\sum_{x=0}^{\infty} p_{x}(\lambda, \alpha)=\frac{1}{N} \sum_{x=0}^{\infty} \frac{(\lambda)_{x, \alpha}}{x !}=\frac{1}{N} e_{\alpha}^{\lambda}(1)=1
$$

and so $N=e_{\alpha}^{\lambda}(1)=(1+\alpha)^{\frac{\lambda}{\alpha}}$. Thus, we state our main definition as follows.

Definition 2. The degenerate Poisson distribution with two parameters $\lambda$ and $\alpha$ is defined by the following formula

$$
p_{x}(\lambda, \alpha)=P(X=x)=e_{\alpha}^{-\lambda}(1) \frac{(\lambda)_{x, \alpha}}{x !} \quad \text { for } x=0,1,2, \ldots
$$

We now investigate some properties of the degenerate Poisson distribution. We firstly give the mean and variance of the mentioned distribution as follows.

Theorem 1. The mean $E(X)$ and the variance $V(X)$ of the degenerate Poisson distribution are given by

$$
E(X)=\frac{\lambda}{1+\alpha} \quad \text { and } \quad V(X)=\frac{\lambda}{(1+\alpha)^{2}}
$$

Proof. By Definition 2, we acquire

$$
\begin{aligned}
\mu_{1} & =E(X)=\sum_{x=0}^{\infty} x p_{x}(\lambda, \alpha)=\sum_{x=0}^{\infty} x e_{\alpha}^{-\lambda}(1) \frac{(\lambda)_{x, \alpha}}{x !} \\
& =e_{\alpha}^{-\lambda}(1) \sum_{x=1}^{\infty} \frac{(\lambda)_{x, \alpha}}{(x-1) !}=e_{\alpha}^{-\lambda}(1) \sum_{x=0}^{\infty} \frac{(\lambda)_{x+1, \alpha}}{x !} \\
& =e_{\alpha}^{-\lambda}(1) \sum_{x=0}^{\infty} \frac{\lambda(\lambda-\alpha)(\lambda-2 \alpha) \cdots(\lambda-(x-1) \alpha)(\lambda-x \alpha)}{x !} \\
& =e_{\alpha}^{-\lambda}(1) \lambda \sum_{x=0}^{\infty} \frac{(\lambda-\alpha)_{x, \alpha}}{x !} \\
& =e_{\alpha}^{-\lambda}(1) \lambda e_{\alpha}^{\lambda-\alpha}(1)=(1+\alpha)^{\frac{-\lambda}{\alpha}} \lambda(1+\alpha)^{\frac{\lambda-\alpha}{\alpha}} \\
& =\frac{\lambda}{1+\alpha},
\end{aligned}
$$


which is the asserted mean in $(2.7)$ and

$$
\begin{aligned}
\mu_{2} & =E\left(X^{2}\right)=\sum_{x=0}^{\infty} x^{2} p_{x}(\lambda, \alpha)=e_{\alpha}^{-\lambda}(1) \sum_{x=0}^{\infty} x^{2} \frac{(\lambda)_{x, \alpha}}{x !} \\
& =e_{\alpha}^{-\lambda}(1) \sum_{x=1}^{\infty}(x-1+1) \frac{(\lambda)_{x, \alpha}}{(x-1) !} \\
& =e_{\alpha}^{-\lambda}(1) \sum_{x=2}^{\infty} \frac{(\lambda)_{x, \alpha}}{(x-2) !}+e_{\alpha}^{-\lambda}(1) \sum_{x=1}^{\infty} \frac{(\lambda)_{x, \alpha}}{(x-1) !} \\
& =e_{\alpha}^{-\lambda}(1) \sum_{x=0}^{\infty} \frac{\lambda(\lambda-\alpha)(\lambda-2 \alpha) \cdots(\lambda-(x-1) \alpha)(\lambda-x \alpha)}{x !}+e_{\alpha}^{-\lambda}(1) \lambda \sum_{x=0}^{\infty} \frac{(\lambda-\alpha)_{x, \alpha}}{x !} \\
& =e_{\alpha}^{-\lambda}(1) \lambda(\lambda-\alpha) \sum_{x=0}^{\infty} \frac{(\lambda-2 \alpha)_{x, \alpha}}{x !}+e_{\alpha}^{-\lambda}(1) \lambda \sum_{x=0}^{\infty} \frac{(\lambda-\alpha)_{x, \alpha}}{x !} \\
& =e_{\alpha}^{-\lambda}(1) \lambda(\lambda-\alpha) e_{\alpha}^{\lambda-2 \alpha}(1)+e_{\alpha}^{-\lambda}(1) \lambda e_{\alpha}^{\lambda-\alpha}(1) \\
& =\frac{\lambda(\lambda-\alpha)}{(1+\alpha)^{2}}+\frac{\lambda}{1+\alpha} \\
& =\frac{\lambda^{2}+\lambda}{(1+\alpha)^{2}} .
\end{aligned}
$$

Therefore, we get the variance

$$
\begin{aligned}
V(X) & =E\left(X^{2}\right)-(E(X))^{2} \\
& =\frac{\lambda^{2}+\lambda}{(1+\alpha)^{2}}-\frac{\lambda^{2}}{(1+\alpha)^{2}} \\
& =\frac{\lambda}{(1+\alpha)^{2}}
\end{aligned}
$$

which is the desired variance in $(2.7)$.

Remark 1. As $\alpha \rightarrow 0$, the mean and variance in (2.7) reduce to the mean and variance for familiar Poisson distribution in (1.3).

The third raw moment (skewness) and fourth raw moment (kurtosis) are given by the following theorem.

Theorem 2. The skewness and the kurtosis for the degenerate Poisson distribution are given below

$$
\mu_{3}=E\left(X^{3}\right)=\frac{\lambda(\lambda-\alpha)(\lambda-2 \alpha)}{(1+\alpha)^{3}}+3 \frac{\lambda(\lambda-\alpha)}{(1+\alpha)^{2}}+\frac{\lambda}{1+\alpha}
$$

and

$$
\mu_{4}=E\left(X^{4}\right)=\frac{\lambda(\lambda-\alpha)(\lambda-2 \alpha)(\lambda-3 \alpha)}{(1+\alpha)^{4}}+6 \frac{\lambda(\lambda-\alpha)(\lambda-2 \alpha)}{(1+\alpha)^{3}}+7 \frac{\lambda(\lambda-\alpha)}{(1+\alpha)^{2}}+\frac{\lambda}{1+\alpha} .
$$

Proof. Utilizing the similar methods used in the proof of the Theorem 1, the proof of Theorem 2 is based on the following expansions

$$
x^{3}=x(x-1)(x-2)+3 x(x-1)+x
$$

and

$$
x^{4}=x(x-1)(x-2)(x-3)+6 x(x-1)(x-2)+7 x(x-1)+x .
$$

So, we omit the proof. 
The moment generating function $E\left(e^{t X}\right)$ of the degenerate Poisson distribution is obtained by the following procedure:

$$
E\left(e^{t X}\right)=e_{\alpha}^{-\lambda}(1) \sum_{x=0}^{\infty} e^{t x} \frac{(\lambda)_{x, \alpha}}{(x-1) !}=\sum_{n=0}^{\infty}\left(e_{\alpha}^{-\lambda}(1) \sum_{x=0}^{\infty} \frac{x^{n}(\lambda)_{x, \alpha}}{(x-1) !} \frac{t^{n}}{n !} .\right.
$$

In usual, the moment generating function of the familiar Poisson distribution coincides the generating function of the usual Bell polynomials, see the formula (1.5). By this motivation, we define the degenerate Bell polynomials, different from Kim's degenerate Bell polynomials in $[9,10]$, as follows:

$$
\sum_{n=0}^{\infty} B_{n, \alpha}(\lambda) \frac{t^{n}}{n !}=e_{\alpha}^{-\lambda}(1) \sum_{x=0}^{\infty} e^{t x} \frac{(\lambda)_{x, \alpha}}{x !} .
$$

In view of (2.10) and (2.11), for $n \geq 0$, the degenerate Bell polynomials are given by the following series:

$$
B_{n, \alpha}(\lambda)=e_{\alpha}^{-\lambda}(1) \sum_{x=0}^{\infty} x^{n} \frac{(\lambda)_{x, \alpha}}{x !} .
$$

Remark 2. As a tends to zero, the formula given in (2.12) reduce to the well known formula for usual Bell polynomials (cf. $[8,9,10])$ :

\begin{tabular}{|c|}
\hline$B_{0, \alpha}(\lambda)=1$ \\
\hline$B_{1, \alpha}(\lambda)=\frac{\lambda}{1+\alpha}$ \\
\hline$B_{2, \alpha}(\lambda)=\frac{\lambda(\lambda-\alpha)}{(1+\alpha)^{2}}+\frac{\lambda}{1+\alpha}$ \\
\hline$B_{3, \alpha}(\lambda)=\frac{\lambda(\lambda-\alpha)(\lambda-2 \alpha)}{(1+\alpha)^{3}}+3 \frac{\lambda(\lambda-\alpha)}{(1+\alpha)^{2}}+\frac{\lambda}{1+\alpha}$ \\
\hline 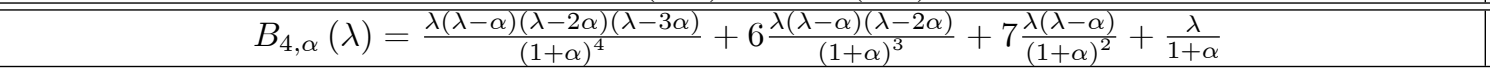 \\
\hline$B_{5, \alpha}(\lambda)=\frac{\lambda(\lambda-\alpha)(\lambda-2 \alpha)(\lambda-3 \alpha)(\lambda-4 \alpha)}{(1+\alpha)^{5}}+10 \frac{\lambda(\lambda-\alpha)(\lambda-2 \alpha)(\lambda-3 \alpha)}{(1+\alpha)^{4}}+25 \frac{\lambda(\lambda-\alpha)(\lambda-2 \alpha)}{(1+\alpha)^{3}}+15 \frac{\lambda(\lambda-\alpha)}{(1+\alpha)^{2}}+\frac{\lambda}{1+\alpha}$ \\
\hline $\begin{array}{c}B_{6, \alpha}(\lambda)=\frac{\lambda(\lambda-\alpha)(\lambda-2 \alpha)(\lambda-3 \alpha)(\lambda-4 \alpha)(\lambda-5 \alpha)}{(1+\alpha)^{6}}+15 \frac{\lambda(\lambda-\alpha)(\lambda-2 \alpha)(\lambda-3 \alpha)(\lambda-4 \alpha)}{(1+\alpha)^{5}} \\
\quad+65 \frac{\lambda(\lambda-\alpha)(\lambda-2 \alpha)(\lambda-3 \alpha)}{(1+\alpha)^{4}}+90 \frac{\lambda(\lambda-\alpha)(\lambda-2 \alpha)}{(1+\alpha)^{3}}+35 \frac{\lambda(\lambda-\alpha)}{(1+\alpha)^{2}}+\frac{\lambda}{1+\alpha} .\end{array}$ \\
\hline
\end{tabular}

$$
B_{n}(\lambda)=e^{-\lambda} \sum_{x=0}^{\infty} x^{n} \frac{\lambda^{x}}{x !} .
$$

With the help of Theorems 1 and 2, the first few degenerate Bell polynomials are

The $\Delta_{\alpha}$ difference operator is defined by (see [3])

$$
\Delta_{\alpha} f(x)=\frac{f(x+\alpha)-f(x)}{\alpha}, \quad \alpha \neq 0 .
$$

Note that as $\alpha$ goes to zero, the $\Delta_{\alpha}$ difference operator reduce to the usual derivative operator:

$$
\lim _{\alpha \rightarrow 0} \Delta_{\alpha} f(x)=\lim _{\alpha \rightarrow 0} \frac{f(x+\alpha)-f(x)}{\alpha}=f^{\prime}(x)=\frac{d f(x)}{d x} .
$$

The following difference rules hold true (cf. [4]):

$$
\Delta_{\alpha}^{k}(x)_{n, \alpha}=\frac{n !}{(n-k) !}(x)_{n-k, \alpha}, \quad 0 \leq k \leq n
$$

and

From (2.14) and (2.15), we obtain

$$
\Delta_{\alpha} e_{\alpha}^{x}(t)=t e_{\alpha}^{x}(t)
$$

$$
\Delta_{\alpha} e_{\alpha}^{-\lambda}(1)=e_{\alpha}^{-\lambda}(1) \text { and } \Delta_{\alpha}(\lambda)_{x, \alpha}=x(\lambda)_{x-1, \alpha} .
$$

We give a product rule of the difference operator in a special case as follows. 
Lemma 1. For $m, x$ being two natural numbers, we then have

$$
\Delta_{\alpha}\left((-\lambda)_{m, \alpha}(\lambda)_{x, \alpha}\right)=m(-\lambda)_{m-1, \alpha}(\lambda)_{x, \alpha}+x(-\lambda+\alpha)_{m, \alpha}(\lambda)_{x-1, \alpha} .
$$

Proof. In terms of (2.13) and (2.16), we attain

$$
\begin{aligned}
\Delta_{\alpha}\left((-\lambda)_{m, \alpha}(\lambda)_{x, \alpha}\right)= & \frac{(-\lambda+\alpha)_{m, \alpha}(\lambda+\alpha)_{x, \alpha}-(-\lambda)_{m, \alpha}(\lambda)_{x, \alpha}}{\alpha} \\
= & \frac{(-\lambda+\alpha)(-\lambda)_{m-1, \alpha}(\lambda+\alpha)(\lambda)_{x-1, \alpha}}{\alpha} \\
& -\frac{(-\lambda)_{m-1, \alpha}(-\lambda-(m-1) \alpha)(\lambda)_{x-1, \alpha}(\lambda-(x-1) \alpha)}{\alpha} \\
= & \frac{(-\lambda)_{m-1, \alpha}(\lambda)_{x-1, \alpha}[(-\lambda+\alpha)(\lambda+\alpha)-(-\lambda-(m-1) \alpha)(\lambda-(x-1) \alpha)]}{\alpha}=(-\lambda)_{m-1, \alpha}(\lambda)_{x-1, \alpha}[m(\lambda+\alpha(-x+1))+x(\alpha-\lambda)] \\
= & m(-\lambda)_{m-1, \alpha}(\lambda)_{x-1, \alpha}(\lambda-(x-1) \alpha)+x(-\lambda+\alpha)(-\lambda)_{m-1, \alpha}(\lambda)_{x-1, \alpha} \\
= & m(-\lambda)_{m-1, \alpha}(\lambda)_{x, \alpha}+x(-\lambda+\alpha)_{m, \alpha}(\lambda)_{x-1, \alpha},
\end{aligned}
$$

which is the claimed result (2.17).

We now improve the properties of the degenerate Poisson distribution. We give the following theorem.

Theorem 3. (Difference Operator Property) For $x \geq 2$, we have

$$
\triangle_{\alpha} p_{x}(\lambda, \alpha)=p_{x}(\lambda, \alpha)+\frac{\lambda}{x-1} p_{x-2}(\lambda-\alpha, \alpha) .
$$

Proof. By means of the Definition 2, the definition of the difference operator (2.13) and Lemma 1, we investigate

$$
\begin{aligned}
\triangle_{\alpha} p_{x}(\lambda, \alpha) & =\triangle_{\alpha}\left(e_{\alpha}^{-\lambda}(1) \frac{(\lambda)_{x, \alpha}}{x !}\right) \\
& =\triangle_{\alpha}\left(\sum_{m=0}^{\infty} \frac{(-\lambda)_{m, \alpha}}{m !} \frac{(\lambda)_{x, \alpha}}{x !}\right) \\
& =\sum_{m=0}^{\infty} \frac{1}{m ! x !} \triangle_{\alpha}\left((-\lambda)_{m, \alpha}(\lambda)_{x, \alpha}\right) \\
(\text { By Lemma 1) } & =\sum_{m=0}^{\infty} \frac{m(-\lambda)_{m-1, \alpha}(\lambda)_{x, \alpha}+x(-\lambda+\alpha)_{m, \alpha}(\lambda)_{x-1, \alpha}}{m ! x !} \\
& =\frac{(\lambda)_{x, \alpha}}{x !} \sum_{m=0}^{\infty} \frac{(-\lambda)_{m-1, \alpha}}{(m-1) !}+\frac{(\lambda)_{x-1, \alpha}}{(x-1) !} \sum_{m=0}^{\infty} \frac{(-\lambda+\alpha)_{m, \alpha}}{m !} \\
& =\frac{(\lambda)_{x, \alpha}}{x !} \sum_{m=0}^{\infty} \frac{(-\lambda)_{m, \alpha}}{m !}+\frac{\lambda}{x-1} \frac{(\lambda-\alpha)_{x-2, \alpha}}{(x-2) !} \sum_{m=0}^{\infty} \frac{(-\lambda+\alpha)_{m, \alpha}}{m !} \\
& =p_{x}(\lambda, \alpha)+\frac{\lambda}{x-1} p_{x-2}(\lambda, \alpha),
\end{aligned}
$$

which means the desired result (2.18). 
In order to get more properties about the degenerate Poisson distribution, we consider that

$$
\begin{aligned}
\frac{(\lambda)_{x, \alpha}}{x !} & =\frac{\lambda(\lambda-\alpha)(\lambda-2 \alpha) \cdots(\lambda-(x-1) \alpha)}{1.2 \ldots(x-1) x} \\
& =\frac{\lambda}{x} \frac{(\lambda-\alpha)(\lambda-2 \alpha) \cdots(\lambda-(x-1) \alpha)}{1.2 \ldots(x-1)} \\
& =\frac{\lambda}{x}(\lambda-\alpha)\left(\frac{\lambda}{2}-\alpha\right) \cdots\left(\frac{\lambda}{x-1}-\alpha\right) \\
& =\frac{\lambda}{x} \prod_{k=1}^{x-1}\left(\frac{\lambda}{k}-\alpha\right) .
\end{aligned}
$$

The unsigned Stirling numbers of the first kind is defined by the rising factorial given below (cf. [1])

$$
x^{\bar{n}}=x(x+1) \cdots(x+n-1)=\sum_{k=0}^{n} S_{1}(n, k) x^{k}
$$

which satisfies the following recurrence relation

$$
S_{1}(n+1, k)=n S_{1}(n, k)+S_{1}(n, k-1)
$$

and the following relations

$$
S_{1}(n, n)=1, S_{1}(n+1,1)=n ! \text { and } S_{1}(n, n-1)=\frac{n(n-1)}{2}
$$

for $n$ being a natural number.

We now give the following theorem.

Theorem 4. For $n \geq 1$, we have

$$
\prod_{k=1}^{n}\left(\frac{\lambda}{k}-\alpha\right)=\frac{1}{n !} \sum_{k=0}^{n} S_{1}(n+1, k+1) \lambda^{k}(-\alpha)^{n-k}
$$

Proof. We prove this theorem by induction: For $n=1$, we get

$$
(\lambda-\alpha)=\frac{1}{1 !} \sum_{k=0}^{1} S_{1}(2, k+1) \lambda^{k}(-\alpha)^{1-k}=S_{1}(2,1)(-\alpha)+S_{1}(2,2) \lambda
$$

which is correct because of the values $S_{1}(2,1)=1=S_{1}(2,2)$. We assume that the formula in (2.23) holds for $n=m$, namely

$$
\prod_{k=1}^{m}\left(\frac{\lambda}{k}-\alpha\right)=\frac{1}{m !} \sum_{k=0}^{m} S_{1}(m+1, k+1) \lambda^{k}(-\alpha)^{m-k}
$$


To prove for $n=m+1$, we compute that

$$
\begin{aligned}
& \prod_{k=1}^{m+1}\left(\frac{\lambda}{k}-\alpha\right)=\left(\frac{\lambda}{m+1}-\alpha\right) \prod_{k=1}^{m}\left(\frac{\lambda}{k}-\alpha\right) \\
& =\left(\frac{\lambda-\alpha(m+1)}{m+1}\right) \frac{1}{m !} \sum_{k=0}^{m} S_{1}(m+1, k+1) \lambda^{k}(-\alpha)^{m-k} \\
& =\frac{1}{(m+1) !} \sum_{k=0}^{m} S_{1}(m+1, k+1) \lambda^{k+1}(-\alpha)^{m-k} \\
& +\frac{1}{m !} \sum_{k=0}^{m} S_{1}(m+1, k+1) \lambda^{k}(-\alpha)^{m+1-k} \\
& =\frac{S_{1}(m+1, m+1) \lambda^{m+1}}{(m+1) !}+\frac{1}{m !} S_{1}(m+1,1)(-\alpha)^{m+1} \\
& +\frac{1}{(m+1) !} \sum_{k=0}^{m-1} S_{1}(m+1, k+1) \lambda^{k+1}(-\alpha)^{m-k}+\frac{1}{m !} \sum_{k=1}^{m} S_{1}(m+1, k+1) \lambda^{k}(-\alpha)^{m+1-k} \\
& =\frac{\lambda^{m+1}}{(m+1) !}+(-\alpha)^{m+1}+\frac{1}{(m+1) !} \sum_{k=1}^{m} S_{1}(m+1, k) \lambda^{k}(-\alpha)^{m+1-k} \\
& +\frac{1}{m !} \sum_{k=1}^{m} S_{1}(m+1, k+1) \lambda^{k}(-\alpha)^{m+1-k} \\
& =\frac{\lambda^{m+1}}{(m+1) !}+(-\alpha)^{m+1} \\
& +\frac{1}{(m+1) !} \sum_{k=1}^{m}\left[S_{1}(m+1, k)+(m+1) S_{1}(m+1, k+1)\right] \lambda^{k}(-\alpha)^{m+1-k} \\
& =\frac{\lambda^{m+1}}{(m+1) !}+(-\alpha)^{m+1}+\frac{1}{(m+1) !} \sum_{k=1}^{m} S_{1}(m+2, k+1) \lambda^{k}(-\alpha)^{m+1-k} \\
& =\frac{1}{(m+1) !} \sum_{k=1}^{m+1} S_{1}(m+2, k+1) \lambda^{k}(-\alpha)^{m+1-k},
\end{aligned}
$$

where we use the formulas (2.21) and (2.22). Therefore, the proof is completed by induction.

By means of Theorem 4 and formula (2.19), we give

$$
(\lambda)_{x, \alpha}=\sum_{k=0}^{x-1} S_{1}(x, k+1) \lambda^{k+1}(-\alpha)^{x-1-k} .
$$

Using (2.24), we rewrite (2.6) and (2.12) with explicit representations as

$$
\begin{aligned}
p_{x}(\lambda, \alpha) & =\frac{e_{\alpha}^{-\lambda}(1)}{x !} \sum_{k=0}^{x-1} S_{1}(x, k+1) \lambda^{k+1}(-\alpha)^{x-1-k} \\
& =\sum_{m=0}^{\infty} \sum_{k=0}^{x-1} \frac{(-\lambda)_{m, \alpha}}{m ! x !} S_{1}(x, k+1) \lambda^{k+1}(-\alpha)^{x-1-k}
\end{aligned}
$$


and

$$
\begin{aligned}
B_{n, \alpha}(\lambda) & =e_{\alpha}^{-\lambda}(1) \sum_{x=0}^{\infty} \frac{x^{n}}{x !} \sum_{k=0}^{x-1} S_{1}(x, k+1) \lambda^{k+1}(-\alpha)^{x-1-k} \\
& =\sum_{m=0}^{\infty} \sum_{x=0}^{\infty} \sum_{k=0}^{x-1} \frac{(-\lambda)_{m, \alpha}}{m ! x !} x^{n} S_{1}(x, k+1) \lambda^{k+1}(-\alpha)^{x-1-k}
\end{aligned}
$$

\section{Conclusion}

In the present paper, the degenerate Poisson distribution covering the degenerate exponential function and the $\alpha$-falling factorial have been defined and investigated. Various properties of the degenerate Poisson distribution such as the first to the fourth raw moments and difference rule have been provided with their proofs. The corresponding moment generating function has been investigated, by which the degenerate Bell polynomials have been considered and their properties have been developed. The results obtained in this paper reduce to the known results for the Poisson distribution and Bell polynomials as $\alpha$ approaches zero.

Conflict of Interest: The authors declare that they have no conflict of interest.

\section{References}

[1] Araci, S.; Duran, U.; Acikgoz, M. On weighted $q$-Daehee polynomials with their applications, Indag. Math. 2019, 30, $365-374$.

[2] Bell, E. T. Exponential polynomials. Ann. Math. 1934, 35, 258-277.

[3] Dattoli, G.; Lorenzutta S.; Cesarano, C. Finite sums and generalized forms of Bernoulli polynomials, Rend. Math. Appl. 1999, 19, 385-391.

[4] Duran, U.; Sadjang, P.N. On Gould-Hopper-based fully degenerate poly-Bernoulli polynomials with a $q$-parameter, Mathematics, 2019, 7, 121.

[5] Evans, M., Hastings, N. A. J., Peacock, B. Statistical distributions, 2nd Edition. John Wiley \& Sons, Inc, New York, 1993.

[6] Frank A.H. Handbook of the Poisson Distribution. New York: John Wiley \& Sons, 1967.

[7] Hermann, R. Generalization of the fractional poisson distribution, 2016, 19, 832-842.

[8] Kim, D.S.; Kim, T. Some identities of Bell polynomials. Sci. China Math. 2015, 58, 2095-2104.

[9] Kim, T.; Kim, D.S. On degenerate Bell numbers and polynomials. RASCAM. 2017, 111, 435-446.

[10] Kim, T.; Kim, D.S.; Dolgy, D.V. On partially degenerate Bell numbers and polynomials, Proc. Jangjeon Math. Soc. 2017, $20,337-345$.

[11] Mahmoudi, E.; Sepahdar, A. Exponentiated Weibull-Poisson distribution: Model, properties and applications, Math. Comp. Simu. 2013, 92, 76-97.

[12] Porwal, S., Dixit, K. K. On Mittag-Leffler type Poisson distribution, Afr. Mat. 2017, 28, 29-34. 